\title{
Fatores técnicos intervenientes na realização do exame de eletrorretinograma multifocal (ERGmf)
}

\author{
Technical factors that influence multifocal electroretinogram(mfERG) recording
}

\author{
Maria Kiyoko Oyamada ${ }^{1}$ \\ Patrícia de Freitas Dotto ${ }^{2}$ \\ Milena Abdalla ${ }^{3}$
}

Trabalho realizado na Clínica de Oftalmologia do Hospital das Clínicas da Faculdade de Medicina da Universidade de São Paulo - USP - São Paulo (SP) - Brasil.

${ }^{1}$ Doutora em Medicina e assistente do Departamento de Oftalmologia do Hospital das Clínicas da Faculdade de Medicina da Universidade de São Paulo - USP - São Paulo (SP) - Brasil.

${ }^{2}$ Pós-graduanda, nível doutorado pela Faculdade de Medicina da USP - São Paulo (SP) - Brasil.

${ }^{3}$ Médica voluntária da Clínica de oftalmologia do Hospital das Clínicas da Faculdade de Medicina da USP São Paulo (SP) - Brasil.

Endereço para correspondência: Maria Kiyoko Oyamada. Rua Matheus Grou, 539 - Apto. 153 - São Paulo (SP) CEP 05415-050

E-mail: kiyoko.ops@terra.com.br

Recebido para publicação em 30.03.2006

Última versão recebida em 31.10.2006

Aprovação em 07.11.2006

\section{RESUMO}

Objetivo: Descrever os principais fatores intervenientes observados durante a aquisição e análise do exame de eletrorretinograma multifocal (ERGmf), que afetam o registro gráfico das ondas obtidas e portanto sua análise. Métodos: Análise dos fatores de erro observados durante a aquisição de 100 exames seqüenciais de eletrorretinograma multifocal, realizados com o Reti System da Roland Consult, no período de maio a julho de 2005. Os exames de eletrorretinograma multifocal foram realizados conforme recomendações internacionais, respeitando-se os parâmetros preestabelecidos do equipamento, com 61 elementos, e ângulo visual de $30^{\circ}$. Todos os pacientes foram submetidos à avaliação oftalmológica completa, excluindo-se aqueles com transparência inadequada de meios, córneas planas e os incapazes de visualizar as linhas de fixação. Resultados: Os fatores intervenientes, que poderiam causar erros na interpretação dos resultados obtidos, observados e corrigidos durante a realização dos exames foram: fixação excêntrica, movimentação dos olhos, contração do orbicular, contração da musculatura cervical, não-correção adequada, não-transparência adequada do eletrodo corneano, deslocamento do eletrodo em geral para baixo, descentralização do suporte da lente corretora, distância inadequada paciente-monitor em pacientes com alta ametropia, altura inadequada dos olhos em relação ao centro da tela, ruído na freqüência de $60 \mathrm{HZ}$ (rede elétrica) e de campo eletromagnético, impedâncias elevadas. No processamento das ondas, a utilização excessiva de filtros para remoção de ruídos dos registros obtidos, pode interferir na análise dos resultados obtidos. Conclusões: Cuidados simplesmente observacionais durante a aquisição dos sinais são de importância significativa para a obtenção de registros com boa morfologia e baixo nível de ruído. Permitindo, por meio de sua pronta correção, a análise correta e adequada da amplitude e do tempo implícito dos picos N1 e P1, fundamentais para a corroboração diagnóstica de lesões ou afecções retinianas.

Descritores: Doenças retinianas/diagnóstico; Eletrorretinografia/métodos; Retina/fisiologia; Potenciais evocados visuais; Erros de diagnóstico

\section{INTRODUĈ̃̃O}

O eletrorretinograma multifocal (ERGmf) é obtido por meio de registro simultâneo das respostas de múltiplas áreas da retina central, utilizando-se estímulos de alta e baixa luminância, apresentados na seqüência denominada sequiência-m (sequiência de extensão ou alcance máximo) em monitor de vídeo. Seu registro gráfico é formado por um conjunto de ondas, obtido por extração matemática de sinais, com picos negativo $\left(\mathrm{N}_{1}\right)$ e positivo $\left(\mathrm{P}_{1}\right)$, semelhantes ao do eletrorretinograma (ERG) de campo total. A análise da 
amplitude e da latência dos picos possibilita a avaliação da função de múltiplas áreas da retina subtendida em aproximadamente $25^{\circ}$ a $30^{\circ}$ radialmente da fóvea. A área equivalente a cada onda obtida varia conforme a sua excentricidade em relação à fóvea e ao número de hexágonos utilizados para a obtenção do resultado.

Componentes lineares ou de primeira ordem (FOK) e não lineares ou de segunda ordem (SOK) podem ser obtidos. A resposta de primeira ordem é a média da resposta obtida de uma área circunscrita da retina, não afetada pela estimulação de áreas circunvizinhas. A de segunda ordem representa a interação temporal entre flashes, quando a apresentação destes se faz em intervalos relativamente menores que o tempo de geração da resposta.

Estudos em humanos demonstram que o pico $\mathrm{N}_{1}$ é gerado com a mesma contribuição celular da onda $a$ e o $\mathrm{P}_{1}$ tem a contribuição dos componentes da onda $b$ e do potencial oscilatório do ERG de campo total de cones ${ }^{(1)}$.

Assim como outros testes diagnósticos, o ERGmf apresenta suas limitações. Sendo uma técnica relativamente nova, ainda necessita de aprimoramentos e de estudos e ensaios para estabelecer variabilidade inter e intraindividual, assim como reprodutibilidade do teste ${ }^{(2)}$. Alguns autores reportam que o limite inferior de normalidade ( $5^{\circ}$ percentil) na área central foi de $27,5 \mathrm{nV} / \mathrm{deg}^{2}$ comparado ao valor médio de $55,8 \mathrm{nV} / \mathrm{deg}^{2}$, e a reprodutibilidade em dois testes foi de $18 \%$ na área central e de $31 \%$ no anel externo $^{(3)}$. Coeficiente de variação transversal de $22 \%$ é relatado em estudos concordantes ${ }^{(4)}$.

A cada dia tem-se aumentado a importância dos testes eletrofisiológicos, quer para análise de função quer para detecção ou confirmação de afecções retinianas. Para tanto a interpretação correta dos registros obtidos são fundamentais na análise do exame, pois pequenas alterações em latência e amplitude podem ser significativas. Para a correta análise dos resultados é necessário o conhecimento do comportamento do resultado obtido diante dos numerosos fatores que podem influenciar o resultado final obtido.

Para avaliar influência dos fatores intervenientes no resultado do ERG multifocal estudamos os principais fatores de erros observados durante a realização dos exames no serviço de Eletrofisiologia do HC FMUSP, utilizando-se o Equipamento da Roland Consult - sistema Retiport.

\section{MÉTODOS}

Observado os fatores intervenientes no resultado do ERGmf, durante a realização de 100 exames seqüenciais realizados no período de 3 meses, no serviço de Eletrofisiologia do HC FMUSP, utilizando-se o sistema Reti System do Equipamento da Roland Consult, procedente de Meinz, Alemanha. Os exames de ERGmf foram realizados conforme recomendações internacionais ${ }^{(5)}$. Respeitando-se os parâmetros pré-estabelecidos do equipamento, com 61 elementos, ângulo visual de $30^{\circ}$ e eletrodo corneano tipo ERGjet. Todos os pacientes foram submetidos à avaliação oftalmológica completa, excluíndo-se aqueles com transparência inadequada de meios, córneas planas e os incapazes de enxergar as linhas de fixação. O exame foi realizado a 23,8 cm da tela; sob dilatação máxima (diâmetro pupilar maior que $8 \mathrm{~mm}$ ), obtida com tropicamida a $1 \%$ e ciclopentolato a $2 \%$; e adição de +3 DE à refração estática obtida. Utilizado colírio anestésico tópico e metilcelulose a 5\% para fixação do eletrodo corneano e realizado leve abrasão da pele na região central da fronte, a $2 \mathrm{~cm}$ do násion e do rebordo temporal das órbitas, para colocação dos eletrodos terra e negativo respectivamente.

\section{RESULTADO}

Os fatores intervenientes, que poderiam causar erros na interpretação dos resultados obtidos, observados e corrigidos durante a realização dos exames foram:

- fixação excêntrica ou inconsistente,

- movimentação dos olhos,

- contração do músculo orbicular e/ou da musculatura cervical,

- posicionamento da pálpebra superior e cílios,

- correção inadequada,

- não transparência adequada do eletrodo corneano,

- deslocamento ou mal posicionamento do eletrodo corneano,

- centralização inadequada do suporte da lente corretora,

- distância inadequada paciente-monitor, em pacientes com alta ametropia,

- altura inadequada dos olhos em relação ao centro da tela,

- ruído na frequiência de $60 \mathrm{HZ}$ (rede elétrica) e de campo eletromagnético,

- impedâncias elevadas,

- uso excessivo de filtros para remoção de ruídos dos registros obtidos.

Na tabela 1 constam alguns dos artefatos observados no registro gráfico final decorrentes destes fatores intervenientes.

\section{DISCUSSÃO}

A heterogeneidade espacial do sistema de cones retinianos em humanos é bastante conhecida, apresentando densidade máxima na fóvea. Além da substancial diminuição da densidade com a excentricidade, há também diversidade quanto à composição celular da via. O ERGmf, desenvolvido por alguns autores $^{(6)}$, permite através da avaliação funcional demonstrar esta heterogeneidade, utilizando-se de abordagens correlacionais cruzadas para obtenção de respostas de vários segmentos focais da retina central. O registro gráfico em $3 \mathrm{D}$ da amplitude das respostas destes segmentos mimetiza o perfil da densidade de cones, com pico central e excentricidade bastante semelhante. Os estímulos, apresentados em monitor de vídeo, são constituídos por padrões hexagonais escalonados conforme sua excentricidade. Durante a estimulação cada hexágono reverte de branco para preto ou vice-versa, em uma seqüência pseu- 
Tabela 1 - Correlação entre fatores técnicos e artefatos observados no registro gráfico do ERGmf

\section{Fatores Intervenientes}

Dilatação pupilar inadequada

Lentes positivas altas

Ângulo visual inadequado

Descentralização do eletrodo corneano ou do suporte da lente corretora

lluminação não uniforme da sala

Posição da pálpebra superior

Fixação excêntrica

Resolução do estímulo

Colaboração do paciente

Ruídos eletromagnéticos

Contração muscular e piscar
Escotoma em anel

Baixa amplitude nos anéis periféricos

Amplitude anômala

Sombra ou depressão localizada

$2 \%$

$3 \%$

$2 \%$

$12 \%$

Excentricidade do pico central

$10 \%$

Flutuação da fixação

Picos irrregulares no registro das ondas

$15 \%$

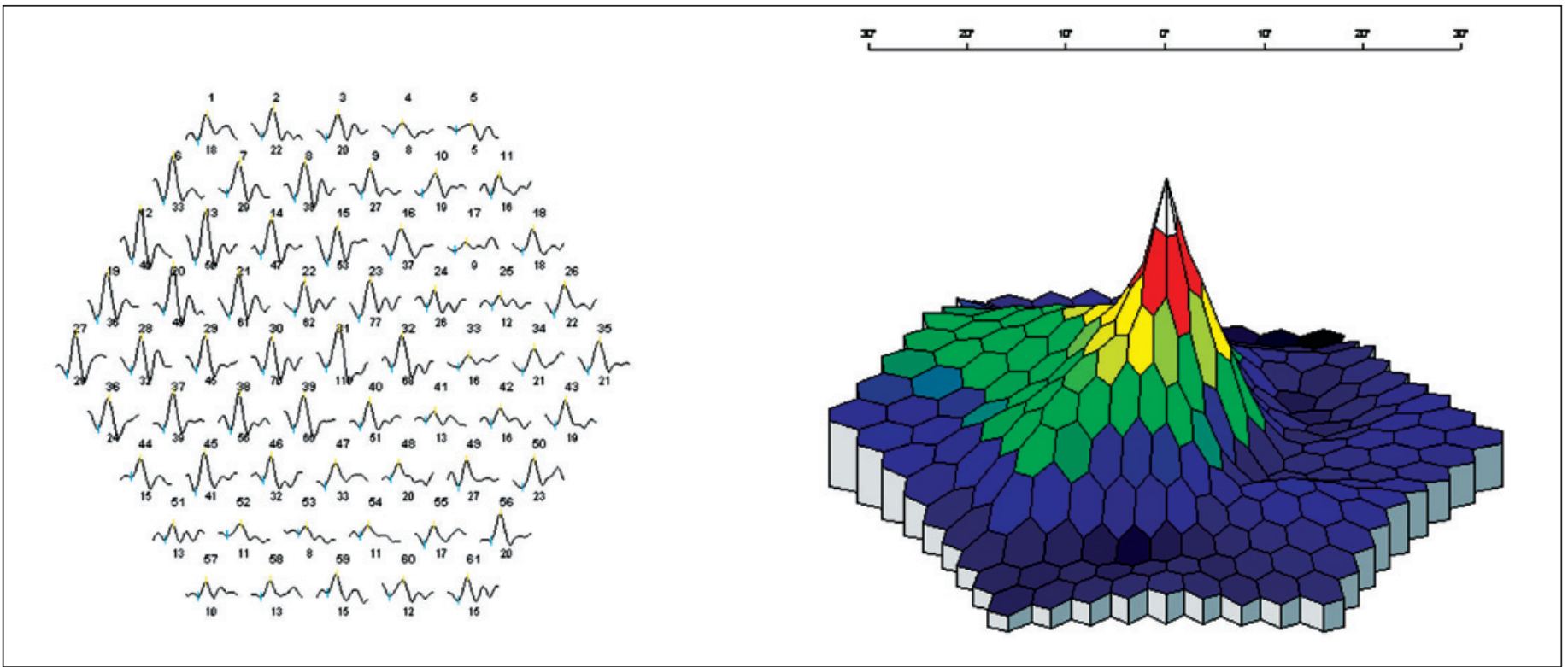

Figura 1 - Sombra localizada devido decentralização do eletrodo corneano
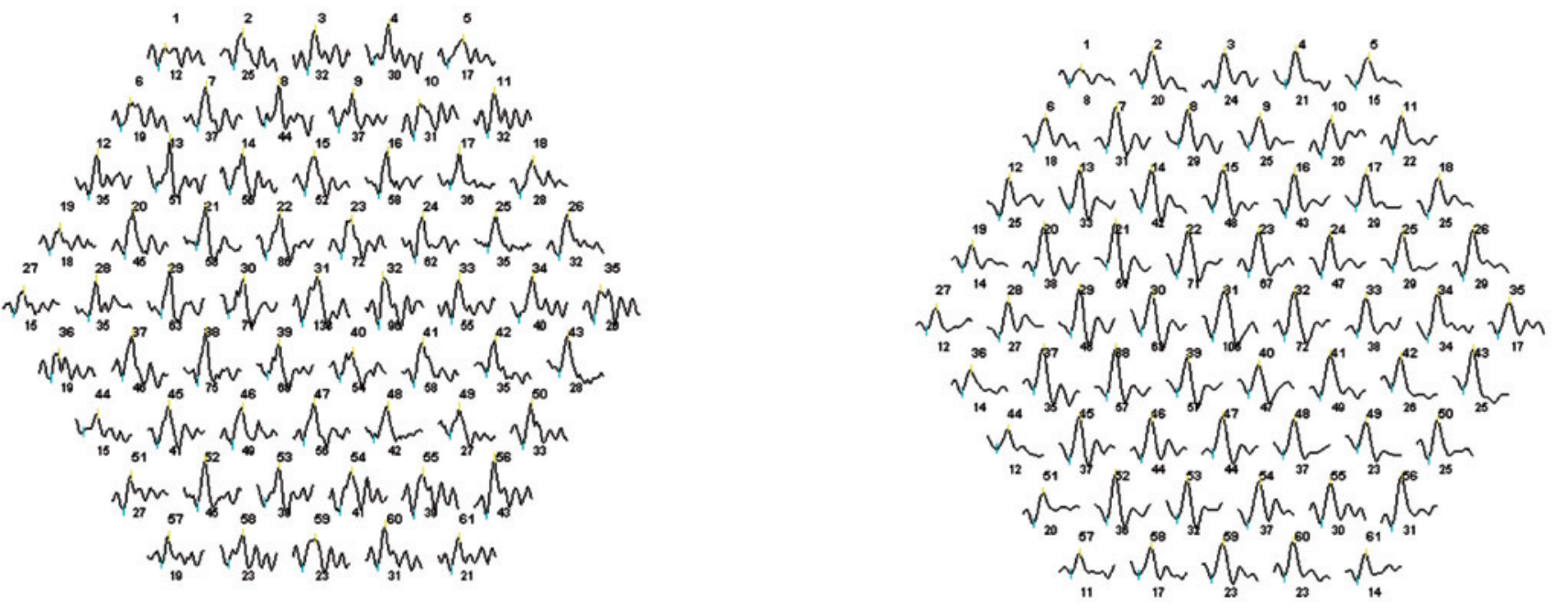

Figura 2 - Redução de amplitude com uso de filtro 
do-randômica, denominada seqüência-m ${ }^{(6)}$. No equipamento desenvolvido pela Roland Consult é utilizado o mesmo padrão de hexágonos, mas apresentados em uma seqüência derivada ao da técnica inicialmente desenvolvida ${ }^{(6)}$.

"Guidelines" tem sido proposto para a realização do exame, mas pesquisas ainda se fazem necessárias para o estabelecimento de protocolos estandardizados ${ }^{(7)}$. O registro do ERGmf requer habilidade e experiência, uma vez que as dificuldades e os desafios na captação dos registros e na análise das respostas do ERGmf são muito maiores do que aqueles envolvidos no eletrorretinograma de campo total.

O método de estimulação é determinante quanto ao ângulo visual, nível de interferência e duração do estímulo e do exame. Respeitando-se as condições de estimulação, propriedades da tela e do sistema de aquisição e registro de dados, considerando-se parâmetros como frequiência, luminância, calibração, tipo e tamanho dos estímulos, frequiência do flicker, contraste e iluminação de fundo, amplificadores e filtros, que apresentam um impacto direto na forma da onda e na distribuição topográfica dos sinais, numerosas outras variáveis podem influenciar a qualidade da onda na resposta final obtida no ERGmf ${ }^{(8)}$.

O sinal do ERGmf sofre o efeito da adaptação e da dispersão da luz na retina circunvizinha. As condições de pré-adaptação e da iluminação ambiente têm influência na resposta obtida no ERG multifocal. No escuro ocorre contaminação da resposta pela luz do estímulo difundida à retina periférica adaptada ao escuro, fato este demonstrado pela significante atenuação dos sinais na mancha cega quando da realização do exame com a sala iluminada. Resultados mais estáveis são obtidas com iluminação ambiente de $1,6 \log \mathrm{cd} / \mathrm{m}^{2(9)}$. Aumento médio de amplitude de $36 \%$ nos componentes negativos e $47 \%$ para componentes positivos foram observados em estudo comparativo, com registros de respostas obtidas em condições de pré-adaptação ao escuro e ao claro, mas com diferença de magnitude significantemente maior na periferia $(58 \%) \mathrm{em}$ relação ao anel central (22\%). Isto reflete as diferenças regionais na retina, assim como a contaminação da resposta pela estimulação da retina adjacente ${ }^{(5)}$. Recomendado pré-adaptação de 15 minutos em sala com iluminação normal, desde que o paciente não tenha sido exposto à claridade excessiva do sol ou realizado exames com luz intensa, como retinografia ou oftalmoscopia indireta.

O tipo de eletrodo, sua colocação, assim como sua limpeza e conservação têm impacto direto na forma das ondas obtidas. O eletrodo de captação deve ter abertura óptica central ou ser suficientemente clara para permitir boa acuidade visual e correção óptica adequada. Eletrodos na forma de lentes de contato devem ser adequadamente higienizados para evitar possíveis alterações de contraste e luminância do estímulo e do aclaramento da retina. Cuidados devem ser redobrados quando da realização de exames seqüenciais como, por exemplo, ERG de campo total seguido da realização de ERGmf, pois o ressecamento da metilcelulose durante os períodos de adaptação pode reduzir a transparência da lente. Os eletrodos de referência e terra devem ser posicionados conforme protocolos padroniza- dos internacionalmente ${ }^{(7)}$. Impedâncias altas, com registro de ruídos, podem decorrer da presença de resíduos ressecados de pasta de contato nestes eletrodos. Recomenda-se a verificação do estado dos fios de condução e da cúpula dos eletrodos a cada exame. A salinização periódica dos eletrodos de referência e terra melhora a condutividade dos mesmos. Um outro fator que altera a impedância é o não preparo adequado da pele. Uma escarificação leve da pele quer com pastas abrasivas ou lixas finas apresentam resultados melhores do que a simples limpeza com álcool.

Depressão da resposta nos anéis periféricos pode decorrer da dilatação inadequada da pupila ou do uso de lentes positivas de dioptrias elevadas. Depressões localizadas ou sombras, isto é depressão em uma parte do gráfico, e elevação no lado oposto, podem resultar da descentração do eletrodo corneano, assim como da lente corretiva ou de iluminação não uniforme da sala. Depressão no anel periférico inferior é observado quando do não posicionamento adequado da pálpebra superior, e pode ser corrigido com a fixação da pálpebra ou posicionando-se o olho do paciente abaixo do centro da tela e inclinando-se ligeiramente o monitor, de forma a manter o paralelismo com o pólo posterior do olho.

Cuidado deve ser redobrado quando do uso de correção óptica que possa provocar magnificação ou redução do estímulo. Isto deve ser compensado com a distância córnea-tela, segundo recomendações de cada fabricante, uma vez que baixa amplitude de resposta pode ser gerada por distância ou ângulo visual inadequado ${ }^{(10)}$.

A condição de fixação constitui um parâmetro importante na integridade dos dados adquiridos, sendo bastante estável para padrões de estímulos cujo elemento central subentenda 2,4 graus. Flutuação de fixação, durante a aquisição do registro, é mais freqüente quando da utilização de estímulos de alta resolução, menores que 2,4 graus $^{(11)}$. A não definição da mancha cega no registro final obtido constitui um indicador de fixação pobre, podendo se associar à depressão central ou aparência inclinada, isto é sinais de baixa amplitude de um lado e de alta amplitude no outro. Registros de qualidade podem ser de difícil obtenção em pacientes com baixa acuidade visual e fixação instável ou excêntrica, gerando respostas falsamente alteradas, conduzindo a interpretação clínica errônea. Fixação estável não significa necessariamente fixação central e acurada, portanto o uso de câmeras de vídeo externa ou de fundo de olho são extremamente úteis ${ }^{(5)}$.

O diâmetro pupilar tem um efeito significante na amplitude e na latência do pico P1 do ERGmf, e deve ser considerada para uma interpretação correta dos resultados. Amplitude e latência de P1 abaixo da média são obtidas com pupilas menores que $7 \mathrm{~mm}$, tanto nos anéis centrais quanto periféricos, sendo recomendado portanto a realização do exame com diâmetro pupilar $\geq 8 \mathrm{~mm}^{(12)}$.

$\mathrm{Na}$ análise e processamento das ondas a utilização de procedimentos como rejeição de artefatos, cálculo de média e promediação de respostas devem ser utilizados com cuidado $^{(8)}$.

A frequiência e a quantidade de ruído durante o registro da 
resposta devem ser observadas. Em nosso meio, picos na frequiência de $60 \mathrm{~Hz}$ decorrem em geral da não filtragem ou do aterramento inadequado do aparelho. Fixação inconsistente, movimentação randômica dos olhos e o piscar excessivo podem produzir sinais irregulares com picos, promovendo a saturação dos amplificadores, direção aberrante ou flutuação nas ondas, com contaminação da resposta de diferentes locos. No gráfico em $3 \mathrm{D}$, o pico central pode ser gerado por somação de artefatos, devendo ser sempre analisado em conjunto com o traçado gráfico do registro de ondas.

Programas disponíveis para remover artefatos de registro, decorrentes do piscar ou da movimentação dos olhos, devem ser aplicados de forma cuidadosa, uma vez que o alisamento e o uso de filtros excessivos reduzem marcadamente a resolução espacial, mascarando pequenas lesões ou induzindo diagnósticos errôneos. As respostas do FOK do ERGmf são constituídas por freqüências abaixo de $65 \mathrm{~Hz}$, com componente espectral principal entre 19 e $47 \mathrm{~Hz}$, enquanto que as do SOK se situam entre 19 e $84 \mathrm{~Hz}$. Estudo conduzido por alguns autores $^{(13)}$, quanto ao impacto do uso de filtro de corte de $50 \mathrm{~Hz}$ em ondas de FOK e SOK, recomenda atenção quanto às alterações na onda devido atenuação dos componentes de maior frequiência do FOK. Atentando-se às atenuação ou reversão de fase, quando da análise dos componentes espectrais principais da resposta do SOK, devido a alterações e distorções das ondas no registro gráfico. O sistema Roland permite a modelação digital da onda obtida, minimizando ou removendo picos em freqüências específicas, resultando numa menor redução de amplitude das ondas.

\section{CONCLUSÃO}

Considerando-se os aspectos discutidos, cuidados simplesmente observacionais durante a aquisição dos sinais são de importância significativa para a obtenção de registros com boa morfologia e baixo nível de ruído. Permitindo a análise correta e adequada da amplitude e do tempo implícito dos N1 e $\mathrm{P} 1$, fundamentais para a corroboração diagnóstica de lesões ou afecções retinianas.

\section{ABSTRACT}

Purpose: To describe the main intervening factors observed during the acquisition and analysis of multifocal electroretinogram (mfERG), that could affect the recorded signal, the obtained responses and their analysis. Methods: The error factors observed during the acquisition of 100 sequential multifocal electroretinogram tests, in the period of May to July, 2005 were analyzed. The examinations of multifocal electroretinogram were carried out in agreement with international guideline recommendations, taking into account the established parameters of equipment, with 61 elements, and visual angle of $30^{\circ}$. All patients had been submitted to complete ophthalmologic examination, excluding those with inadequate transparency of ways, flat corneas and those incapable to visualize a central fixation target. Results: The intervening factors, that could cause artifacts and errors in the interpretation of the obtained results, observed and corrected during the acquisition of multifocal electroretinogram were: eccentric or poor fixation, eye movements, orbicular contraction, cervical muscle contraction, inadequate transparency of the corneal electrode, electrode displacement, lens support decentralization, inadequate viewing distance in patients with high refractive errors, eye height inadequacy in relation to the center of the screen, electrical noise in $60 \mathrm{HZ}$ frequency and electromagnetic field and high impedances. Excessive use of filters in wave processing for noise and artifact removal can interfere with the analysis of the obtained results. Conclusion: Simple observational cares during signal acquisition are of significant importance to ensure accuracy of the topographical electroretinogram information, good waveform morphology and low noise level register. Through their ready correction, the correct and adjusted analysis of the amplitude and of implicit time of peaks N1, P1 and N2 is reliable. Digital smooth system with specific frequency peak removal supply waves with more defined morphology with lower amplitude reduction than other available resources.

Keywords: Retinal diseases/diagnosis; Electroretinography/ methods; Retina/physiology; Evoked potentials, visual; Diagnostic errors

\section{REFERÊNCIAS}

1. Hood DC, Seiple W, Holopigian K, Greenstein VA. A comparison of the components of the multifocal an full-field ERGs. Vis Neurosci. 1997;14(3):533-44.

2. Meigen T, Friedrich A. [The reproducibility of multifocal ERG recordings]. Ophthalmologe. 2002;99(9):713-8. German.

3. Parks S, Keating D, Williamson TH, Evans AL, Elliott AT, Jay JL. Functional imaging of the retina using multifocal electroretinography: a control study. Br J Ophthtalmol. 1996;80(9):831-4.

4. Aoyagi K, Kimura Y, Isono H, Akiyama H. [Reproducibility and wave analysis of multifocal electroretinography]. Nippon Gankkai Zashi. 1998;102(5):340-7. Japanese.

5. Sutter EE e Tran D. The field topography of ERG components in man - I. The photopic luminance response. Vision Res. 1992;32(3):433-46.

6. Marmor MF, Hood DC, Keating D, Kondo M, Seeliger MW, Miyake Y; International Society for Clinical Electrophysiology of Vision. Guidelines for basic multifocal eletroretinography (mfERG). Doc Ophthalmol. 2003;106(3):338.

7. Keating D, Parks S, Evans AL. Technical aspects of multifocal ERG recording. Doc Ophthalmol. 2000;100(2-3):77-98.

8. Chappelow AV, Marmor MF. Effects of pre-adaptation conditions and ambient room lighting on the multifocal ERG. Doc Ophthalmol. 2002;105(1):23-31.

9. Kondo M, Miyake Y, Piao CH, Tanikawa A, Horiguchi M, Terasaki H. Amplitude increase of the multifocal electroretinogram during light adaptation. Invest Ophthalmol Sci. 1999;40(11):2633-7.

10. Palmowski AM, Berninger T, Allgayer R, Andrielis H, Heinemann-Vernaleken B, Rudolph G. Effects of refractive blur on the multifocal electroretinogram. Doc Ophthalmol. 1999;99(1):41-54.

11. Chisholm JA, Keating D, Parks S, Evans AL. The impact of fixation on the multifocal electroretinogram. Doc Ophthalmol. 2001;102(2):131-9.

12. Gonzalez P, Parks S, Dolan F, Keating D. The effects of pupil size on the multifocal electroretinogram. Doc Ophthalmol. 2004;109:67-72

13. Bock M, Gerth C, Lorenz B. Impact of notch filter use on waveforms of Firstand Second-Order-Kernel responses from multifocal ERGs. Doc Ophthalmol. 2000;101(3):195-210. 